\title{
Nesting an incompressible-flow code within a compressible-flow code: a two-dimensional study
}

\author{
Michael A. Sprague ${ }^{\mathrm{a}, *}$, Ignas Satkauskas ${ }^{\mathrm{a}, \mathrm{b}}$ \\ ${ }^{a}$ Computational Science Center, National Renewable Energy Laboratory, 15013 Denver \\ West Parkway, Golden, CO 80401, USA \\ ${ }^{b}$ Department of Applied Mathematics, University of Colorado, 526 UCB, Boulder, CO \\ 80309, USA
}

\begin{abstract}
We consider numerical algorithms appropriate for one- and two-way coupling between meso-scale and micro-scale fluid-dynamics codes for wind energy computing. At the meso-scale is a numerical weather-prediction code, which is typically based on the compressible-flow Euler equations. At the micro-scale, surrounding one or more wind turbines, is a computational fluid dynamics code, which is typically based on the incompressible-flow NavierStokes equations. When calculating short-duration flow around wind turbines, one-way coupling is sufficient, where the meso-scale computational model drives the micro-scale model. However, in long-duration simulations involving large wind farms, the influence of the wind farm on the meso-scale weather may no longer be insignificant and two-way coupling is warranted. In this study, we focus on a simple two-dimensional system, for which our goal is to devise one- and two-way coupling algorithms that can effectively transport a vortex propagating in laminar flow from one domain to the other.
\end{abstract}

*Corresponding author. E-mail address: Michael.A.Sprague@nrel.gov 
Two coupling schemes and their numerical implementation are described: partial-boundary coupling and projection coupling. In the former, the microscale-domain boundary is decomposed, based on the meso-scale solution, into sections corresponding to inflow and outflow. The micro-scale model has Dirichlet- and Neumann-type boundary conditions on these sections, respectively. In projection coupling, the meso-scale solution is projected onto the incompressible-flow solution space in the micro-scale domain, from which Dirichlet-type boundary conditions are derived. In these simulations, the uncoupled meso-scale solution is taken as the reference, and the best coupling method is that which produces solutions that deviate the least from the reference. In one-way coupling, under a simple two-dimensional laminarflow test case, partial-boundary coupling was more effective than projection coupling. However, in two-way coupling, projection coupling was the best performer.

Keywords: wind energy, multi-scale, model coupling, computational fluid dynamics, numerical weather prediction

\section{Introduction}

Computational models present opportunities for reducing the cost of wind energy by enabling scientists and engineers to better predict the associated complicated multi-physics and to better optimize turbine designs and wind farm layouts. While it is clear that the show-stopping engineering problems surrounding a single wind turbine have largely been solved (after all, wind turbines are being built and installed), large-scale deployment of wind farms, composed of hundreds of wake-interacting wind turbines, faces daunting chal- 
lenges due to poorly understood flow physics. For example [1], observed power loss can be $20-30 \%$ of that predicted in operational wind farms due to poorly understood turbine-wake interactions in large wind farm arrays $[2,3]$, and turbines within wind farms experience significantly higher failure rates compared to isolated turbines due to uncertain aerodynamic loading [4]. Further, it is unclear in what ways large wind farms will affect regional weather, which is an important issue considering that wind farms are often centered in agricultural regions. Understanding and overcoming these challenges will be aided by high-fidelity computational models that account for interaction with the local weather system.

The physical systems governing production and extraction of wind energy have relevant time and length scales spanning many orders of magnitude [5]. Physically validated mathematical models and software are established at each uncoupled scale; however, no single model can treat all relevant scales. For example, meso-scale atmospheric dynamics are described well by solutions to the compressible Euler equations (with appropriate physics models), whereas turbine-vicinity (micro-scale) fluid dynamics are well described by solutions to the incompressible Navier-Stokes (NS) equations (with an appropriate subgrid-turbulence model). While the need for multi-model multi-scale coupled systems is clear, there is no obvious path for efficient and accurate coupling for holistic simulation across relevant scales.

In this paper, we are focused on numerical methods appropriate for coupling numerical weather prediction (NWP) codes (meso-scale) with turbinelocal computational fluid dynamics (CFD) codes (micro-scale). Further, we are interested in codes for micro-scale flow that have unstructured-grid ca- 
pability allowing accurate simulation over complex topography. We focus on the Weather Research and Forecasting (WRF) code [6] as our target meso-scale code and an incompressible-flow CFD code like OpenFOAM [7] as our target micro-scale code. Coupling such codes has an abundance of challenges. First, as mentioned above, there is an inherent mismatch of the mathematical models. The WRF model is, at its core, built on the compressible inviscid Euler equations. At the micro-scale, the turbine-vicinity model is built on the incompressible viscous Navier-Stokes equations. Thus, acoustic waves exist in the meso-scale model, while the micro-scale model is acoustically rigid. Second, there are numerical-model mismatches between WRF and, say, an OpenFOAM implementation: (i) the spatial and temporal grids will not match at their interface, and WRF is discretized in the vertical direction in pressure coordinates - the spatial grid moves vertically with variation in pressure; (ii) WRF employs an explicit time integrator, whereas OpenFOAM employs a semi-implicit time integrator; (iii) WRF is spatially discretized with finite differences on a structured grid, whereas OpenFOAM is discretized with finite volumes on, in general, an unstructured grid.

While there have been limited efforts to two-way couple WRF and an incompressible-flow CFD code (see, e.g., [8]), there is no evidence of successful interactive coupling. Alternatively, there has been more success in one-way coupling, where a meso-scale NWP model is used to drive an incompressibleflow CFD model. Li et al. [9] used the RAMS NWP model to drive a FLUENT incompressible-flow CFD simulation in a Reynolds averaged NavierStokes (RANS) framework. WRF-calculated velocity and temperature fields were used on all lateral and top boundaries in the FLUENT model. Boutan- 
ios et al. [10] performed OpenFOAM RANS simulations where the boundary conditions were taken from a WRF simulation. Zajaczkowski et al. [11] performed a preliminary study where WRF results were used as boundary conditions for an AcuSolve [12] CFD simulation, again with a RANS model. WRF velocities were used as inflow boundary conditions on two sides of the CFD box; other boundaries had simple outflow boundary conditions. A Newtonian-relaxation data assimilation technique was used in transferring WRF data to AcuSolve. Recently, Churchfield et al. [13] used WRF and WRF-LES (large-eddy simulation), to drive an OpenFOAM incompressibleflow CFD simulation with a standard Smagorinsky LES model in their Simulator fOr Wind Farm Applications (SOWFA). WRF data were used as OpenFOAM boundary conditions, where the mean-flow direction was into the OpenFOAM domain; outflow boundary conditions were used elsewhere. Gopalan et al. [16] and Sitaraman et al. [14] used WRF data to one-way drive a wind farm compressible-flow CFD simulation in the HELIOS computational platform [15] (with RANS and detached-eddy-simulation techniques) where the full rotors were resolved. WRF-HELIOS coupling was accomplished via the meso-scale/micro-scale interface (MMCI [16]), which is a python-based infrastructure. Yang et al. [17] used WRF at the meso-scale to drive their Virtual Wind Simulator, which is a LES framework for simulating incompressible turbulent flow around wind turbines in complex terrain. Further discussion of coupling NWP meso-scale models and CFD micro-scale models for wind-engineering applications can be found in Yamada and Koike [18]. Peet and Lele [19] examined methods for coupling a compressible-flow code with either a low-Mach-number-flow or incompressible-flow code. In Pete and 
Lele's preferred approach, domains interacted at their boundaries; variables were transferred between codes through a simple interpolation. In their analysis of coupling between compressible- and incompressible-flow-models, they restricted simulations to where the underlying test flow was incompressible. Peet and Lele [20] applied their approach to two-dimensional LES of film cooling where a low-Mach-number-flow code was coupled to a compressibleflow code.

There have been successful efforts to one- and two-way couple a nestedgrid large-eddy-simulation inside a meso-scale simulation grid, where the same underlying mathematical model is used at both scales (e.g., compressible flow at both scales). Sullivan et al. [21] examined LES of planetaryboundary-layer (PBL) incompressible flows where a refined grid resided in a coarse grid. One- and two-way coupling was examined. Moeng et al. [22] examined two-way grid nesting for LES PBL compressible flows in the WRF model. Recently, Mirocha et al. [23, 24] showed that the use of one-waycoupled nested grids in WRF LES simulations can improve accuracy. Harris and Durran [25] used an idealized one-dimensional model to study one- and two-way grid nesting.

In this paper, we focus on a simple two-dimensional system that captures large-scale flow characteristics of the meso- and micro-scale systems. The two-dimensional compressible inviscid Euler equations (with no additional physics models) constitute our meso-scale model and the two-dimensional incompressible Navier-Stokes equations (with no turbulence modeling) constitute our micro-scale model. Numerical solution of these models mimics that of WRF and OpenFOAM, i.e., finite-differences spatial discretization 
and explicit time integration for the meso-scale code and finite-volume spatial discretization and semi-implicit time integration for the micro-scale code. With these codes, we test two coupling schemes in one- and two-way coupling. This work is meant to be a foundation-creating step towards the development of robust, accurate, and efficient coupling algorithms appropriate for production computing. Toward that end we examine our coupling schemes with a simple propagating vortex and quantify the errors introduced due to model coupling and the discrepancies between the underlying models. With an effective coupling strategy that can propagate simple flows between models with high fidelity in a physically meaningful way, we can then move towards solving the challenging issues associated with, e.g., the transfer of sub-grid turbulent energy and turbulent-flow structures.

The paper is organized as follows. In Section 2 we describe our twodimensional model system and our coupling algorithms. In Section 3 we describe our numerical models. In Section 4 we present numerical results for one- and two-way coupling for the propagation of a laminar vortex. Section 5 is our conclusion.

\section{Formulation and implementation}

\subsection{Test system}

Figure 1 shows our idealized two-dimensional test system, which consists of two domains: $(i)$ an inner square domain, denoted $\Omega_{\mathrm{NS}}$, with boundary denoted $\partial \Omega_{\mathrm{NS}}$, where we are interested in solutions to the NS equations, and (ii) a larger, partially coincident square domain, denoted $\Omega_{\mathrm{E}}\left(\Omega_{\mathrm{NS}} \subset \Omega_{\mathrm{E}}\right)$ with external boundary denoted $\partial \Omega_{\mathrm{E}}$, where we are interested in solutions 
to the Euler equations. Position (nondimensionalized with a reference length $\left.\ell^{*}\right)$ is denoted $\mathbf{x}=(x, y) \in \Omega_{\mathrm{E}}$, and the coordinate-system origin is at the center of the aligned square domains $\Omega_{\mathrm{NS}}$ and $\Omega_{\mathrm{E}}$.

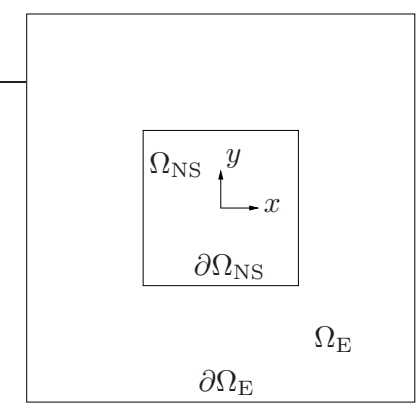

Figure 1: Schematic showing the two-dimensional domains: $\Omega_{\mathrm{E}}$ (meso-scale; compressible Euler) and $\Omega_{\mathrm{NS}}$ (micro-scale; incompressible Navier-Stokes).

The NS nondimensional momentum and continuity equations for twodimensional incompressible flow are

$$
\begin{aligned}
\frac{\partial \mathbf{u}_{\mathrm{NS}}}{\partial t}+\mathbf{u}_{\mathrm{NS}} \cdot \nabla \mathbf{u}_{\mathrm{NS}} & =-\frac{1}{\rho_{\mathrm{NS}}} \nabla p_{\mathrm{NS}}+R e^{-1} \nabla^{2} \mathbf{u}_{\mathrm{NS}}, \\
\nabla \cdot \mathbf{u}_{\mathrm{NS}} & =0
\end{aligned}
$$

respectively, for $\mathbf{x} \in \Omega_{\mathrm{NS}}$, where $t$ is time, and NS-domain-specific quantities are velocity $\mathbf{u}_{\mathrm{NS}}(t, \mathbf{x})=\left(u_{\mathrm{NS}}, v_{\mathrm{NS}}\right)$, density $\rho_{\mathrm{NS}}$ (constant), and pressure $p_{\mathrm{NS}}(t, \mathbf{x})$. Our system is nondimensionalized with reference velocity magnitude $u^{*}$, length $\ell^{*}$, density $\rho^{*}$, dynamic viscosity $\mu^{*}$, and pressure $p^{*}=u^{* 2} \rho^{*}$. The Reynolds number is $R e=u^{*} \ell^{*} \rho^{*} / \mu^{*}$. The reference density is taken as that of the NS-domain, and thus $\rho_{\mathrm{NS}} \equiv 1$. The velocity field has initial conditions $\mathbf{u}_{\mathrm{NS}}(0, \mathbf{x})=\mathbf{u}_{\mathrm{NS}}^{0}(\mathbf{x})$. Boundary conditions will depend on the coupling strategy, and are discussed in subsequent sections. 
The Euler equations (nondimensionalized as above) for momentum and mass conservation, and the state equations for inviscid, isentropic, compressible flow are

$$
\begin{gathered}
\frac{\partial \mathbf{u}_{\mathrm{E}}}{\partial t}+\mathbf{u}_{\mathrm{E}} \cdot \nabla \mathbf{u}_{\mathrm{E}}=-\frac{1}{\rho_{\mathrm{E}}} \nabla p_{\mathrm{E}}, \\
\frac{\partial \rho_{\mathrm{E}}}{\partial t}+\nabla \cdot\left(\rho_{\mathrm{E}} \mathbf{u}_{\mathrm{E}}\right)=0 \\
p_{\mathrm{E}}=\rho_{\mathrm{E}}^{\gamma},
\end{gathered}
$$

respectively, for $\mathbf{x} \in \Omega_{\mathrm{E}}$, where Euler-domain-specific quantities are velocity $\mathbf{u}_{\mathrm{E}}(t, \mathbf{x})=\left(u_{\mathrm{E}}, v_{\mathrm{E}}\right)$, density $\rho_{\mathrm{E}}(t, \mathbf{x})$, and pressure $p_{\mathrm{E}}(t, \mathbf{x})$, and where $\gamma$ is the ratio of specific heats. Periodic boundary conditions are assumed along $\partial \Omega_{\mathrm{E}}$, and the solution is initialized as $\left(\rho_{\mathrm{E}}(0, \mathbf{x}), \mathbf{u}_{\mathrm{E}}(0, \mathbf{x})\right)=\left(\rho_{\mathrm{E}}^{0}(\mathbf{x}), \mathbf{u}_{\mathrm{E}}^{0}(\mathbf{x})\right)$ for $\mathbf{x} \in \Omega_{\mathrm{E}}$.

\subsection{One-way coupling}

An inherent challenge in coupling the meso- and micro-scale flows is that $\nabla \cdot\left(\rho_{\mathrm{NS}} \mathbf{u}_{\mathrm{NS}}\right)=0$ (incompressible flow), while $\nabla \cdot\left(\rho_{\mathrm{E}} \mathbf{u}_{\mathrm{E}}\right) \neq 0$ (compressible flow). Thus, the simple one-way-coupling boundary condition (BC) defined by $\mathbf{u}_{\mathrm{NS}}=\mathbf{u}_{\mathrm{E}}, \forall \mathbf{x} \in \partial \Omega_{\mathrm{NS}}$, is, in general, incompatible with the incompressibility constraint on $\mathbf{u}_{\mathrm{NS}}$. We discuss below two coupling schemes for one-way coupling, where the meso-scale flow drives micro-scale flow: $(i)$ partial-boundary coupling and (ii) projection coupling. For one-way coupling we assume that the meso-scale solution $\left(\mathbf{u}_{\mathrm{E}}, \rho_{\mathrm{E}}\right)$ is known in $\Omega_{\mathrm{E}}$ and for $0 \leq t \leq t_{f}$, where $t_{f}$ is the final simulated time. 


\subsubsection{Partial-boundary coupling}

As an example, consider the case where the meso-scale flow direction is everywhere South-West, i.e., $\mathbf{u}_{\mathrm{E}} \geq \mathbf{0}$. A reasonable one-way coupling approach for the NS domain would be to have "inflow" conditions on the south and west boundaries, and "outflow" conditions on the east and north boundaries. For general flow conditions, inflow and outflow boundary domains are determined as

$$
\begin{aligned}
\partial \Omega_{\mathrm{NS}, \text { in }} & =\left\{\mathbf{x} \mid \mathbf{x} \in \partial \Omega_{\mathrm{NS}}, \mathbf{u}_{\mathrm{E}} \cdot \mathbf{n} \leq 0\right\}, \\
\partial \Omega_{\mathrm{NS}, \text { out }} & =\left\{\mathbf{x} \mid \mathbf{x} \in \partial \Omega_{\mathrm{NS}}, \mathbf{u}_{\mathrm{E}} \cdot \mathbf{n}>0\right\},
\end{aligned}
$$

respectively, where $\mathbf{n}$ is the positive-out normal of $\partial \Omega_{\mathrm{NS}}$. NS-domain boundary conditions are then

$$
\begin{gathered}
\mathbf{u}_{\mathrm{NS}}=\mathbf{F}, \quad \forall \mathbf{x} \in \partial \Omega_{\mathrm{NS}, \text { in }}, \\
\frac{\partial \mathbf{u}_{\mathrm{NS}}}{\partial n}=\mathbf{0}, \quad \forall \mathbf{x} \in \partial \Omega_{\mathrm{NS}, \text { out }}, \\
\frac{\partial p_{\mathrm{NS}}}{\partial n}=0, \quad \forall \mathbf{x} \in \partial \Omega_{\mathrm{NS}},
\end{gathered}
$$

where $\frac{\partial(\cdot)}{\partial n}$ denotes differentiation in the normal-out direction of the boundary, and $\mathbf{F}=\mathbf{F}\left(\mathbf{u}_{\mathrm{E}}, \rho_{\mathrm{E}}, \rho_{\mathrm{NS}}\right)$ is a function to be specified. So long as some portion of the boundary $\partial \Omega_{\mathrm{NS}}$ has an outflow condition, the problem is well posed - the velocity field along the boundary segment where there is an outflow condition will be such that the flow in the NS domain can satisfy the incompressibility constraint, $\nabla \cdot \mathbf{u}_{\mathrm{NS}}=0, \forall \mathbf{x} \in \Omega_{\mathrm{NS}}$. Variations on this basic approach were employed by Zajaczkowski et al. [11] and Churchfield et al. [13]. 
In regard to our choice of $\mathbf{F}$, we take guidance from kinetic energy, as that is of primary interest in wind energy. If we take

$$
\mathbf{F}=\sqrt{\frac{\rho_{\mathrm{E}}}{\rho_{\mathrm{NS}}}} \mathbf{u}_{\mathrm{E}},
$$

then

$$
\mathbf{u}_{\mathrm{NS}}=\mathbf{u}_{\mathrm{E}} \sqrt{\frac{\rho_{\mathrm{E}}}{\rho_{\mathrm{NS}}}}, \quad \forall \mathbf{x} \in \partial \Omega_{\mathrm{NS}, \mathrm{in}},
$$

and the kinetic-energy density is continuous across the domain inflow boundaries, i.e.,

$$
\frac{\rho_{\mathrm{NS}}}{2} \mathbf{u}_{\mathrm{NS}} \cdot \mathbf{u}_{\mathrm{NS}}=\frac{\rho_{\mathrm{E}}}{2} \mathbf{u}_{\mathrm{E}} \cdot \mathbf{u}_{\mathrm{E}}, \quad \forall \mathbf{x} \in \partial \Omega_{\mathrm{NS}, \text { in }} .
$$

While $\rho_{\mathrm{NS}} \equiv 1$ in this nondimensionalization, it is maintained in the above equations for clarity. Under reasonable coupling scenarios, we expect $\rho_{\mathrm{NS}} \approx$ $\rho_{\mathrm{E}}$, which provides $\mathbf{u}_{\mathrm{NS}} \approx \mathbf{u}_{\mathrm{E}}$.

An inherent limitation in this approach is that if the NS-domain inflow and outflow boundaries are determined as described above, then $\partial \Omega_{\mathrm{NS}, \text { in }}$ and $\partial \Omega_{\mathrm{NS}, \text { out }}$ will, in general, change with time in realistic flow conditions. Under this approach, we expect that the transition between inflow and outflow BCs, i.e, Dirichlet- and Neumann-type BCs, respectively, could occur on any one or more of the four straight boundaries in $\partial \Omega_{\mathrm{NS}}$; such a transition on a straight boundary may complicate numerical solution. One-way partialboundary coupling is appealing, however, for its algorithmic and numericalimplementation simplicity.

Numerical implementation of partial-boundary coupling is straightforward. The meso-scale solution is known at discrete times and spatial locations; values may be interpolated to the micro-scale time and space grids for determination of the inflow domain $\partial \Omega_{\mathrm{NS} \text {,in }}$ and the boundary conditions. 
However, the choice of an initial condition $\mathbf{u}_{\mathrm{NS}}^{0}$ is not clear. One might take $\mathbf{u}_{\mathrm{NS}}^{0}=\sqrt{\frac{\rho_{\mathrm{E}}^{0}}{\rho_{\mathrm{NS}}}} \mathbf{u}_{\mathrm{E}}^{0}$ for $\mathbf{x} \in \Omega_{\mathrm{NS}}$. Depending on the magnitude of $\nabla \cdot \mathbf{u}_{\mathrm{NS}}^{0}$, however, the NS solver may be challenged. An alternative is to project the Euler-domain initial condition onto an incompressible solution as described in the next subsection.

\subsubsection{Projection coupling}

We propose here a coupling scheme that does not have the flow-direction challenge of the partial-boundary coupling scheme described in the previous subsection. We desire a function $\mathbf{G}\left(\mathbf{u}_{\mathrm{E}}, \rho_{\mathrm{E}}, \rho_{\mathrm{NS}}\right)$ such that the Dirichlet-type $\mathrm{BC}$ for the entire NS domain is

$$
\mathbf{u}_{\mathrm{NS}}=\mathbf{G}\left(\mathbf{u}_{\mathrm{E}}, \rho_{\mathrm{E}}, \rho_{\mathrm{NS}}\right), \quad \forall \mathbf{x} \in \partial \Omega_{\mathrm{NS}}
$$

Again, we assume that the Euler-domain solution $\left(\mathbf{u}_{\mathrm{E}}, \rho_{\mathrm{E}}\right)$ is known for $\mathbf{x} \in$ $\Omega_{\mathrm{E}}, 0 \leq t \leq t_{f}$, and it is used to drive the solution $\mathbf{u}_{\mathrm{NS}}$ for $\mathbf{x} \in \Omega_{\mathrm{NS}}$. We employ the concept of projection through Lagrange multipliers. Given $\left(\mathbf{u}_{\mathrm{E}}, \rho_{\mathrm{E}}\right)$, we wish to find a Lagrange-multiplier scalar field $\lambda(t, \mathbf{x})$ such that

$$
\nabla \cdot\left(\rho_{\mathrm{E}} \mathbf{u}_{\mathrm{E}}-\nabla \lambda\right)=0, \quad \forall \mathbf{x} \in \Omega_{\mathrm{NS}}
$$

which, assuming homogeneous boundary conditions, leads to a Poisson problem in $\lambda$ : find $\lambda$ such that

$$
\begin{aligned}
\nabla^{2} \lambda & =\nabla \cdot\left(\rho_{\mathrm{E}} \mathbf{u}_{\mathrm{E}}\right), \quad \forall \mathbf{x} \in \Omega_{\mathrm{NS}} \\
\lambda & =0, \quad \forall \mathbf{x} \in \partial \Omega_{\mathrm{NS}} .
\end{aligned}
$$

An associated incompressible velocity field is

$$
\widetilde{\mathbf{u}}=\frac{1}{\rho_{\mathrm{NS}}}\left(\rho_{\mathrm{E}} \mathbf{u}_{\mathrm{E}}-\nabla \lambda\right), \quad \forall \mathbf{x} \in \Omega_{\mathrm{NS}}
$$


and the boundary conditions for the NS domain are taken as

$$
\mathbf{u}_{\mathrm{NS}}=\widetilde{\mathbf{u}}, \quad \frac{\partial p_{\mathrm{NS}}}{\partial n}=0, \quad \forall \mathbf{x} \in \partial \Omega_{\mathrm{NS}} .
$$

We refer to this approach as one-way projection coupling.

In regard to the numerical implementation of one-way projection coupling, it is more involved than that for partial-boundary coupling, but is nonetheless straightforward. The calculation of the field $\lambda$ is closely related to the calculation of the pressure field in standard incompressible-flow CFD solvers (as required to maintain a divergence-free flow). The Euler flow field can be interpolated to the NS grid (at a given time) and the existing CFDcode Poisson solver can be used to calculate $\lambda$ for all $\mathbf{x} \in \Omega_{\mathrm{NS}}$ at each time step.

\subsection{Two-way coupling}

We consider here two-way coupling of the solutions for the Euler and Navier-Stokes domains. Loose coupling is employed, where numerical models for each system, i.e., each partition, can be time-updated in a staggered procedure [26] (i.e., sequential integration). Because our meso-scale solver mimics the WRF solver, as described above, it is not practical to have a mesoscale domain, say $\Omega_{\mathrm{E}}^{\prime}$, that has a "hole" in it such that $\Omega_{\mathrm{E}}^{\prime} \cup \Omega_{\mathrm{NS}}=\emptyset$ and where solutions would interact through their boundaries. As such, and as in the one-way coupling described above, $\Omega_{\mathrm{NS}} \subset \Omega_{\mathrm{E}}$. Two-way coupling described here resembles the two-way nested-grid coupling in WRF-alone calculations (see $\$ 5$ in [6]), where a local fine grid resides (i.e., is nested) in a larger coarse grid. In our approach, however, the local fine grid is rather a micro-scale model that may or may not have a finer grid than the meso-scale model. 
In two-way coupling of nested grids in WRF-alone calculations, numerical solutions are "smoothed" at the interface through "relaxation layers," which introduce diffusion. In our approach, however, we introduce no such layers.

Our description of two-way coupling is simplified by examining discretetime solutions. We assume micro- and meso-scale solutions are known at $t=t^{n}$, and we wish to advance all solutions to $t^{n+1}=t^{n}+\Delta t$. Here, we assume that both codes (meso and micro) are time integrated in lock step, i.e., both have the same $\Delta t$. Our time-update procedure can be summarized as follows:

1. Time advance the meso-scale solution over one time step explicitly from known information.

2. Using boundary conditions extracted from the meso-scale solution, time advance the micro-scale solution semi-implicitly.

3. In the overlapping domain, overwrite the meso-scale solution with one derived from the micro-scale solution.

Detailed descriptions of two-way partial-boundary and projection coupling are below.

\subsubsection{Partial-boundary coupling}

Two-way partial-boundary coupling is a straight-forward extension of oneway partial-boundary coupling. Assuming solutions in the Euler and NS domains are known at time $t=t^{n}$ (i.e., $\mathbf{u}_{\mathrm{E}}^{n}, \rho_{\mathrm{E}}^{n}, p_{\mathrm{E}}^{n}, \mathbf{u}_{\mathrm{NS}}^{n}$, and $\left.p_{\mathrm{NS}}^{n}\right)$, the update of meso- and micro-scale solutions from $t^{n}$ to $t^{n+1}$ proceeds as follows:

1. The meso-scale solution is advanced (explicitly) to time-station $t^{n+1}$, yielding a predicted solution for all $\mathbf{x} \in \Omega_{\mathrm{E}}$, denoted 
$\left(\mathbf{u}_{\mathrm{E}}^{p, n+1}, \rho_{\mathrm{E}}^{p, n+1}, p_{\mathrm{E}}^{p, n+1}\right)$.

2. The inflow and outflow boundary domains, $\partial \Omega_{\mathrm{NS} \text {,in }}$ and $\partial \Omega_{\mathrm{NS} \text {,out }}$, respectively, are determined from the predicted solutions via Eqs. (6) and (7).

3. The micro-scale solution is advanced (semi-implicitly) to $t^{n+1}$, with boundary conditions

$$
\begin{aligned}
\mathbf{u}_{\mathrm{NS}}^{n+1} & =\mathbf{u}_{\mathrm{E}}^{p, n+1} \sqrt{\frac{\rho_{\mathrm{E}}^{p, n+1}}{\rho_{\mathrm{NS}}}}, \quad \forall \mathbf{x} \in \partial \Omega_{\mathrm{NS}, \text { in }}, \\
\frac{\partial \mathbf{u}_{\mathrm{NS}}^{n+1}}{\partial n} & =\mathbf{0}, \quad \forall \mathbf{x} \in \partial \Omega_{\mathrm{NS}, \text { out }}, \\
\frac{\partial p_{\mathrm{NS}}^{n+1}}{\partial n} & =0, \quad \forall \mathbf{x} \in \partial \Omega_{\mathrm{NS}},
\end{aligned}
$$

to yield $\mathbf{u}_{\mathrm{NS}}^{n+1}, p_{\mathrm{NS}}^{n+1}, \forall \mathbf{x} \in \Omega_{\mathrm{NS}}$.

4. Finalize (correct) the meso-scale solution as

$$
\begin{aligned}
& \mathbf{u}_{\mathrm{E}}^{n+1}=\mathbf{u}_{\mathrm{NS}}^{n+1} \sqrt{\frac{\rho_{\mathrm{NS}}}{\rho_{\mathrm{E}}^{p, n+1}}}, \quad \forall \mathbf{x} \in \Omega_{\mathrm{NS}}, \\
& \mathbf{u}_{\mathrm{E}}^{n+1}=\mathbf{u}_{\mathrm{E}}^{p, n+1}, \quad \forall \mathbf{x} \in \Omega_{\mathrm{E}} \backslash \Omega_{\mathrm{NS}}, \\
& \rho_{\mathrm{E}}^{n+1}=\rho_{\mathrm{E}}^{p, n+1}, \quad p_{\mathrm{E}}^{n+1}=p_{\mathrm{E}}^{p, n+1}, \quad \forall \mathbf{x} \in \Omega_{\mathrm{E}} .
\end{aligned}
$$

\subsubsection{Projection coupling}

In two-way projection coupling, assuming solutions in the Euler and NS domains are known at time-station $t=t^{n}\left(\mathbf{u}_{\mathrm{E}}^{n}, \rho_{\mathrm{E}}^{n}, p_{\mathrm{E}}^{n}, \mathbf{u}_{\mathrm{NS}}^{n}, p_{\mathrm{NS}}^{n}\right)$, the update of meso- and micro-scale solutions proceeds as follows:

1. The meso-scale solution is advanced (explicitly) to time-station $t^{n+1}$, yielding a predicted solution for all $\mathbf{x} \in \Omega_{\mathrm{E}}$, denoted $\left(\mathbf{u}_{\mathrm{E}}^{p, n+1}, \rho_{\mathrm{E}}^{p, n+1}, p_{\mathrm{E}}^{p, n+1}\right)$. 
2. The predicted meso-scale solution is interpolated onto the micro-scale grid for all $\mathrm{x} \in \Omega_{\mathrm{NS}}$, and the Lagrange-multiplier problem (Eqs. (16) and $(17))$ is solved at $t^{n+1}$, yielding $\lambda^{n+1}$, and the projected incompressible solution $\widetilde{\mathbf{u}}^{n+1}$.

3. The micro-scale solution is advanced (semi-implicitly) to $t^{n+1}$, with boundary conditions

$$
\mathbf{u}_{\mathrm{NS}}^{n+1}=\widetilde{\mathbf{u}}^{n+1}, \quad \forall \mathbf{x} \in \partial \Omega_{\mathrm{NS}}
$$

to yield $\mathbf{u}_{\mathrm{NS}}^{n+1}, p_{\mathrm{NS}}^{n+1}, \forall \mathbf{x} \in \Omega_{\mathrm{NS}}$.

4. Finalize (correct) the meso-scale solution as

$$
\begin{aligned}
& \mathbf{u}_{\mathrm{E}}^{n+1}=\left(\rho_{\mathrm{NS}} \mathbf{u}_{\mathrm{NS}}^{n+1}+\nabla \lambda^{n+1}\right) / \rho_{\mathrm{E}}^{n+1}, \quad \forall \mathbf{x} \in \Omega_{\mathrm{NS}}, \\
& \mathbf{u}_{\mathrm{E}}^{n+1}=\mathbf{u}_{\mathrm{E}}^{p, n+1}, \quad \forall \mathbf{x} \in \Omega_{\mathrm{E}} \backslash \Omega_{\mathrm{NS}}, \\
& \rho_{\mathrm{E}}^{n+1}=\rho_{\mathrm{E}}^{p, n+1}, \quad p_{\mathrm{E}}^{n+1}=p_{\mathrm{E}}^{p, n+1}, \quad \forall \mathbf{x} \in \Omega_{\mathrm{E}} .
\end{aligned}
$$

In essence, we are "reverse" projecting the micro-scale solution back onto the meso-scale solution.

\section{Numerical methods}

\subsection{Meso-scale code}

Our meso-scale code is a simplified two-dimensional representation of the WRF code [6]. Details regarding the numerical discretization closely follow the description in [27] in a two-dimensional (horizontal) extension of the onedimensional equations presented; we give an overview here. The domain $\Omega_{\mathrm{E}}$ is discretized in $x$ and $y$ with uniform grid size $\Delta_{\mathrm{E}}$. Data are stored in a Cgrid staggered form; velocities are calculated on cell faces while pressure and 
density are calculated at cell centers. Time integration is accomplished with a time-splitting method where the "slow" advective modes are propagated with an explicit third-order Runge-Kutta (RK3) scheme, while the "fast" acoustic modes are propagated over substeps with the forward-backward scheme of Mesinger [28]. Fifth-order-accurate spatial discretization, which is inherently diffusive, is employed for flux-divergence terms.

\subsection{Micro-scale code}

Our micro-scale code is a two-dimensional structured-mesh cell-centered finite-volume code; it was designed to mimic the OpenFOAM finite-volume solver used by Churchfield et al. [3] in large-scale simulations of wind-plant aerodynamics. Oscillations associated with pressure-velocity decoupling are avoided with a Rhie-Chow-like [29] approach for velocity-flux interpolation at cell faces. For other quantities, face values are linearly interpolated from cellcenter values and second-order centered-differences are used for derivatives. Time integration is accomplished with a pressure-implicit splitting operation (PISO) scheme [30]. This is a predictor-corrector approach; in our system we use one predictor and two correctors per time step. The domain $\Omega_{\mathrm{NS}}$ is discretized with square cells, each with side length $\Delta_{\mathrm{NS}}$.

\subsection{Model coupling}

For simplicity, our models are discretized with the same cell sizes $\left(\Delta_{\mathrm{E}}=\right.$ $\left.\Delta_{\mathrm{NS}}\right)$, and the micro-scale equations are time integrated with a step size equal to the advective time step in the meso-scale code. However, as de-

scribed above, the meso-scale code takes additional substeps. In transferring velocities from the meso-scale code to the micro-scale code, cell-face-centered 
velocities are taken directly from the former as boundary conditions for the latter because the grids are matching; there is no interpolation. However, because velocities are stored at cell centers in the micro-scale code, velocities must be interpolated to cell faces in transferring them to the meso-scale code. In practice, it is unlikely that the spatial or temporal grids would be matching. Implementation of the methods proposed here would require additional spatial and/or temporal interpolation in transferring data between meshes.

\section{Results and discussion}

\subsection{Test-problem description}

For evaluation of the one- and two-way coupling schemes described above, we examine the advection of an isentropic vortex [31,32], which is an exact solution to the two-dimensional compressible Euler equations. This is an attractive benchmark problem for examining flow artifacts associated with model coupling; the Euler-domain solution (in the absence of coupling or numerical errors $), \mathbf{u}_{\mathrm{E}}(t, \mathbf{x})$ and $\rho_{\mathrm{E}}(t, \mathbf{x})$, is simply the initial solution $\left(\mathbf{u}_{\mathrm{E}}^{0}, \rho_{\mathrm{E}}^{0}\right)$ translated in the direction of the mean flow $\mathbf{u}_{\infty}$ over a distance $\left(t-t_{0}\right)\left|\mathbf{u}_{\infty}\right|$. In an unbounded domain, the initial isentropic vortex is defined as

$$
\begin{aligned}
& u_{\mathrm{E}}^{0}(x, y)=u_{\infty}-\frac{\alpha y}{2 \pi} \exp \left[\eta\left(1-r^{2}\right)\right], \\
& v_{\mathrm{E}}^{0}(x, y)=v_{\infty}+\frac{\alpha x}{2 \pi} \exp \left[\eta\left(1-r^{2}\right)\right], \\
& \rho_{\mathrm{E}}^{0}(x, y)=\left\{T_{\infty}-\frac{(\gamma-1) \alpha^{2}}{16 \eta \gamma \pi^{2}} \exp \left[2 \eta\left(1-r^{2}\right)\right]\right\}^{\frac{1}{(\gamma-1)}},
\end{aligned}
$$

where the vortex (nondimensional) parameters are taken as follows: $u_{\infty}=$

$v_{\infty}=T_{\infty}=1, r=\sqrt{\left(x-x_{0}\right)^{2}+\left(y-y_{0}\right)^{2}}, x_{0}=y_{0}=-5, \alpha=2$, and $\eta=1$. 
Our problem domains are $\Omega_{\mathrm{E}}=\{(x, y) \mid-10<x<10,-10<y<10\}$ and $\Omega_{\mathrm{NS}}=\{(x, y) \mid-2.5<x<2.5,-2.5<y<2.5\}$. Figure 2 shows the perturbation-velocity magnitude $\left|\mathbf{u}_{\mathrm{E}}-\mathbf{u}_{\infty}\right|$ at $t=5$ over $\Omega_{\mathrm{E}} ; \Omega_{\mathrm{NS}}$ is outlined. For the numerical experiments described below, $\gamma=1.4$ and the NavierStokes equations were solved with $R e=100$, which is orders of magnitude smaller than that in atmospheric flows. However, for our two-dimensional tests, we are interested in the performance of coupling algorithms under laminar flow.

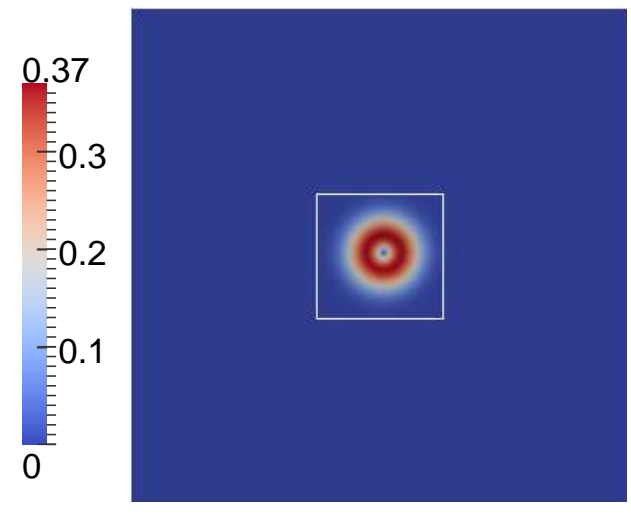

Figure 2: Perturbation-velocity magnitude $\left|\mathbf{u}_{\mathrm{E}}-\mathbf{u}_{\infty}\right|$ in $\Omega_{\mathrm{E}}$ for the isentropic vortex at $t=5$; the domain $\Omega_{\mathrm{NS}}$ is outlined.

\subsection{One-way coupling}

We consider first one-way coupling, where the Euler solution is driving the NS solution. Because the NS-domain is free of obstacles (e.g., wind turbines), we desire that the flow field closely mimics that of the driving meso-scale flow, though the NS-domain flow certainly will deviate from the Euler-domain solution due to model differences (e.g., viscous dissipation). 
As a test of our coupling schemes, we take the numerically calculated, uncoupled time-dependent Euler (meso-scale) solution, denoted $\mathbf{u}_{\mathrm{E}}^{\mathrm{UC}}$, as the benchmark. Of course, in one-way coupling, $\mathbf{u}_{\mathrm{E}}^{\mathrm{UC}}=\mathbf{u}_{\mathrm{E}}$. We compare within $\Omega_{\mathrm{NS}}$ the numerical solutions under partial-boundary and projection coupling. Time integration was performed with a Courant number of $C=0.5$, based on the maximum advective velocity of 1.78 , which is taken from the known isentropic-vortex solution. For the Euler-domain code, two acoustic substeps were taken per advective time step. For the numerical solution discussed below, $\Omega_{\mathrm{E}}$ and $\Omega_{\mathrm{NS}}$ were discretized with $400 \times 400$ and $100 \times 100$ grid points, respectively, resulting in $\Delta_{\mathrm{E}}=\Delta_{\mathrm{NS}}=0.05$. For coarser grids, the numerical solutions were prone to oscillatory instabilities arising in the North-East corner. Of the two one-way coupling methods, the oscillations were more pronounced for projection coupling.

Figure 3 shows the perturbation-velocity magnitude $\left|\mathbf{u}_{\mathrm{NS}}-\mathbf{u}_{\infty}\right|$ in $\Omega_{\mathrm{NS}}$ at $t=5$. Results are shown for the two coupling schemes and we see that the vortex structure is largely maintained in both cases. Figure 4 shows normalized magnitude of the velocity deviation, $D\left(\mathbf{u}_{\mathrm{NS}}\right)$, in $\Omega_{\mathrm{NS}}$, where

$$
D(\mathbf{u})=\frac{\left|\mathbf{u}_{\mathrm{E}}^{\mathrm{UC}}-\mathbf{u}\right|}{\max _{\mathbf{x} \in \Omega_{\mathrm{E}}}\left|\mathbf{u}_{\mathrm{E}}^{\mathrm{UC}}-\mathbf{u}_{\infty}\right|}
$$

We expect $D\left(\mathbf{u}_{\mathrm{NS}}\right)>0$ for any coupled flow regardless of coupling method due, as described above, to the difference between the underlying models. However, since the NS domain is free of obstacles, we desire to minimize deviation. Here, we see that partial-boundary and projection coupling yield a maximum deviation of about 0.22 and 0.30 , respectively. Thus, at this time instance, partial-boundary coupling appears to introduce less deviation from the driving flow compared to projection coupling. For reference, Fig. 5 shows 
the Lagrange-multiplier field at $t=5$ from which boundary conditions were derived for the projection coupling. Considering the meso-scale flow field (Fig. 2) and Eq. (18), the meso-scale flow deviates from being divergencefree in proportion to the gradient of the Lagrange-multiplier field shown in Fig. 5.

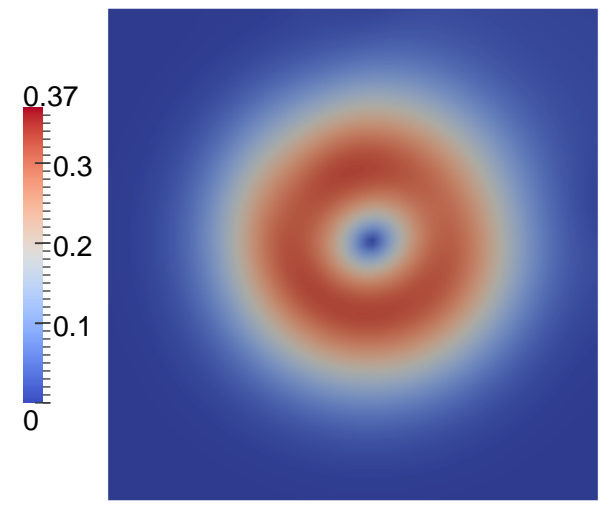

(a) Partial-boundary

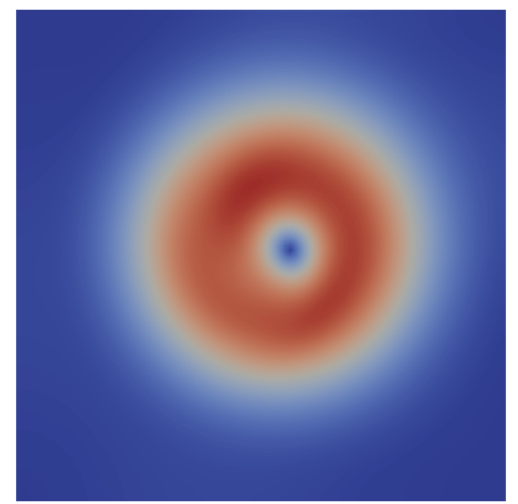

(b) Projection

Figure 3: Perturbation-velocity magnitude $\left|\mathbf{u}_{\mathrm{NS}}-\mathbf{u}_{\infty}\right|$ in $\Omega_{\mathrm{NS}}$ at $t=5$ for (a) partialboundary and (b) projection one-way coupling.

Figure 6 shows the time evolution of the root-mean-square (RMS) of the normalized velocity deviation calculated over $\Omega_{\mathrm{NS}},\|D\|_{\mathrm{RMS}}^{\Omega_{\mathrm{NS}}}$, where

$$
\|D\|_{\mathrm{RMS}}^{\Omega}=\left[\frac{\int_{\Omega} D^{2} \mathrm{~d} \Omega}{\int_{\Omega} \mathrm{d} \Omega}\right]^{\frac{1}{2}} .
$$

For $2.5 \lesssim t \lesssim 7.5$, again, partial-boundary coupling introduced significantly less deviation from the driving flow compared to projection coupling. However, for $t \gtrsim 7.5$, projection coupling restores the NS-domain flow to that of the Euler domain, whereas partial-boundary coupling exhibits an extended period of deviation as the diffused vortex exits the NS domain through the outflow boundary. 


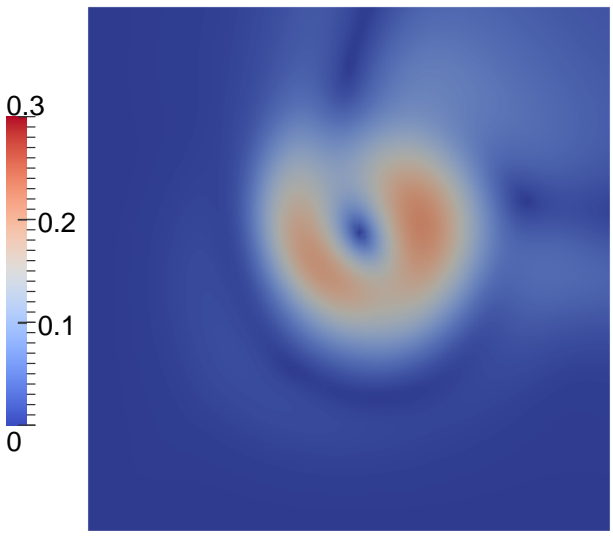

(a) Partial-boundary

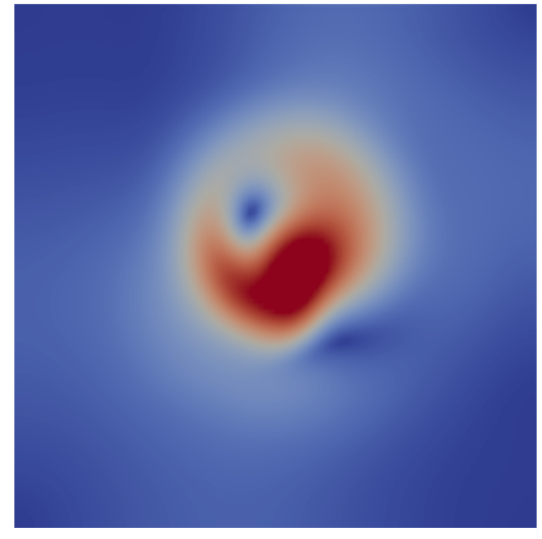

(b) Projection

Figure 4: Normalized magnitude of the velocity deviation, $D\left(\mathbf{u}_{\mathrm{NS}}\right)$, in $\Omega_{\mathrm{NS}}$ at $t=5$ for (a) partial-boundary and (b) projection one-way coupling.

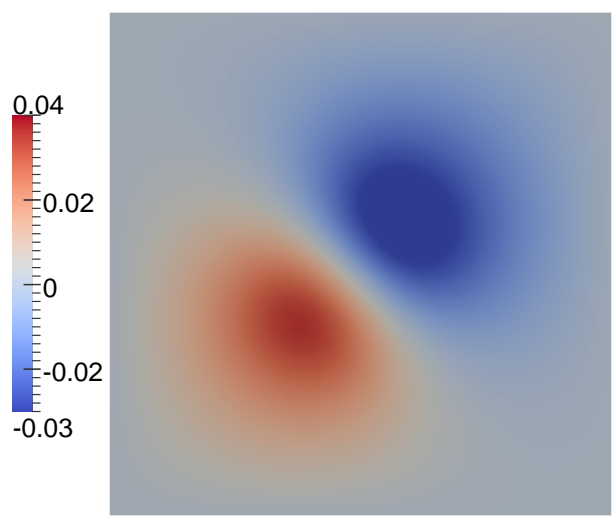

Figure 5: Lagrange-multiplier field in $\Omega_{\mathrm{NS}}$ at $t=5$ for one-way projection coupling.

Since this work is directed at computational modeling of wind energy extraction, the kinetic energy of the flow is an important consideration. Figure 7 shows the evolution of the eddy kinetic energy, $\operatorname{EKE}\left(\Omega_{\mathrm{NS}}, \mathbf{u}, \rho\right)$, calculated over the domain $\Omega_{\mathrm{NS}}$, where

$$
\operatorname{EKE}(\Omega, \mathbf{u}, \rho)=\frac{1}{2} \int_{\Omega} \rho\left(\mathbf{u}-\mathbf{u}_{\infty}\right)^{2} \mathrm{~d} \Omega
$$




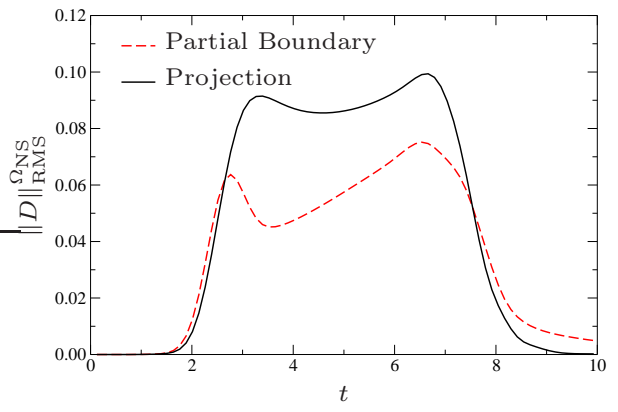

Figure 6: RMS of the normalized velocity deviation calculated over $\Omega_{\mathrm{NS}},\left\|D\left(\mathbf{u}_{\mathrm{NS}}\right)\right\|_{\mathrm{RMS}}^{\Omega_{\mathrm{NS}}}$, as a function of time under partial-boundary and projection one-way coupling.

for the uncoupled flow (i.e., $\mathbf{u}_{\mathrm{E}}^{\mathrm{UC}}$ ) and for the NS-domain flow under partialboundary and projection coupling strategies. As expected, the EKE in the NS domain is less than that in the uncoupled Euler domain due to viscous dissipation. The two coupling strategies produce solutions that exhibit similar EKE in $0<t<10$, with projection coupling yielding slightly better performance.

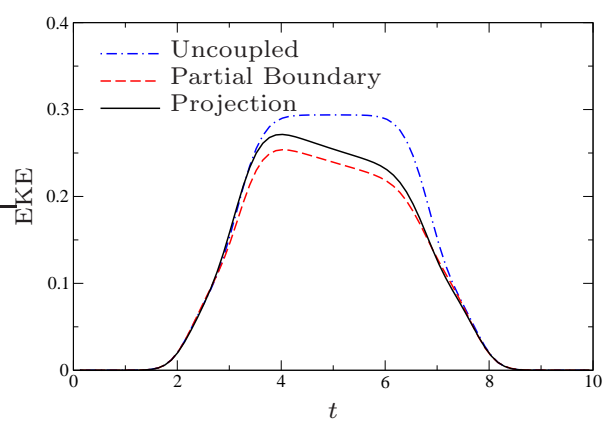

Figure 7: Eddy kinetic energy in $\Omega_{\mathrm{NS}}$ for the uncoupled Euler-domain solution $\left(\rho_{\mathrm{E}}^{\mathrm{UC}}, \mathbf{u}_{\mathrm{E}}^{\mathrm{UC}}\right)$ and the NS-domain solutions $\left(\rho_{\mathrm{NS}}, \mathbf{u}_{\mathrm{NS}}\right)$ as a function of time under under partialboundary and projection one-way coupling. 


\subsection{Two-way coupling}

We examine here two-way coupling and focus on the meso-scale flow and its variation due to coupling with the micro-scale flow. The simulation parameters were the same as those used in one-way coupling. Figure 8 shows the perturbation velocity magnitude in the Euler domain at $t=5$ and $t=10$ for the two coupling methods. The location of the NS domain is outlined. At these instances, the vortex in projection coupling better represents the vortex in the reference solution (c.f., Fig. 2). However, both methods successfully passed the vortex coherently through the domains, and simulations under two-way projection coupling did not exhibit the sensitivity to numerical instabilities as was observed in the one-way case. The superior performance of two-way projection coupling is more clearly shown by the normalized magnitude of the velocity deviation shown in Fig. 9. Here, in partial-boundary coupling, we see the scattering of acoustic waves of significant amplitude; errors are broadly distributed about the vortex center, and these deviations increase with time. However, the solutions produced with projection coupling show much less deviation, and the magnitude of the acoustic waves is much reduced. The magnitude of the deviation in the vortex core appears to increase with time.

Figure 10(a) shows the normalized RMS of the velocity deviation, $\left\|D\left(\mathbf{u}_{\mathrm{E}}\right)\right\|_{\mathrm{RMS}}^{\Omega_{\mathrm{E}}}$, for $0 \leq t \leq 10$ in two-way coupling. The deviation under projection coupling for all times is roughly half that under partial-boundary coupling. Figure $10(\mathrm{~b})$ shows $\left\|D\left(\mathbf{u}_{\mathrm{NS}}\right)\right\|_{\mathrm{RMS}}^{\Omega_{\mathrm{NS}}}$, where we have included oneway-coupling histories from Figure 6. We see that two-way projection cou-

pling introduces the least deviation by a significant margin; two-way coupling 


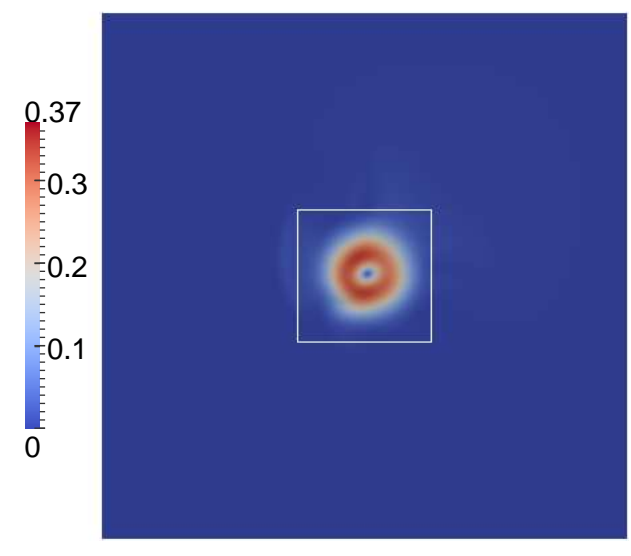

(a) Partial-boundary; $t=5$

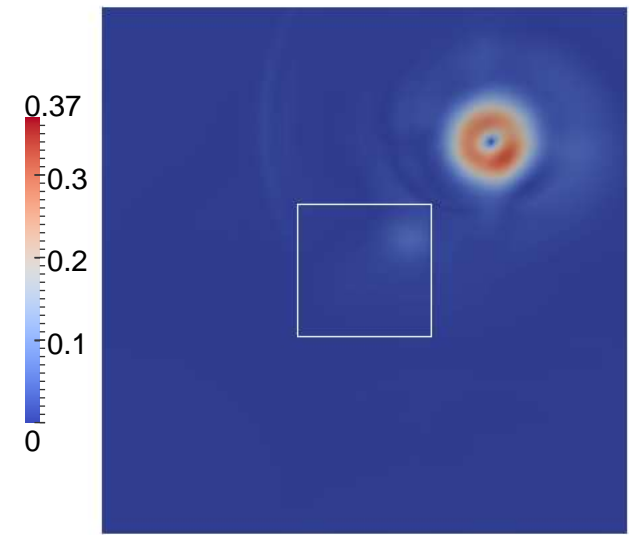

(c) Partial-boundary; $t=10$

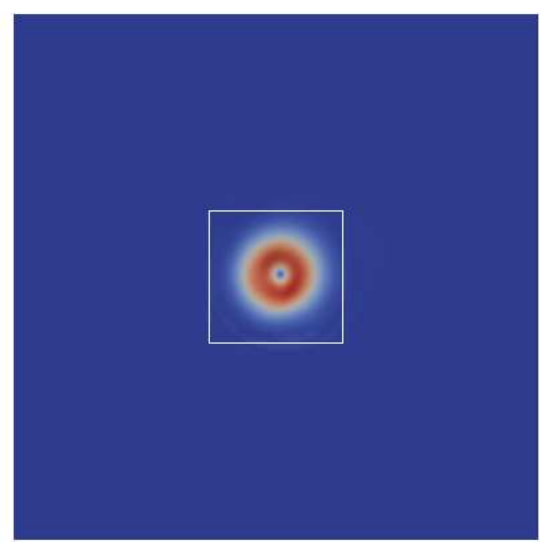

(b) Projection; $t=5$

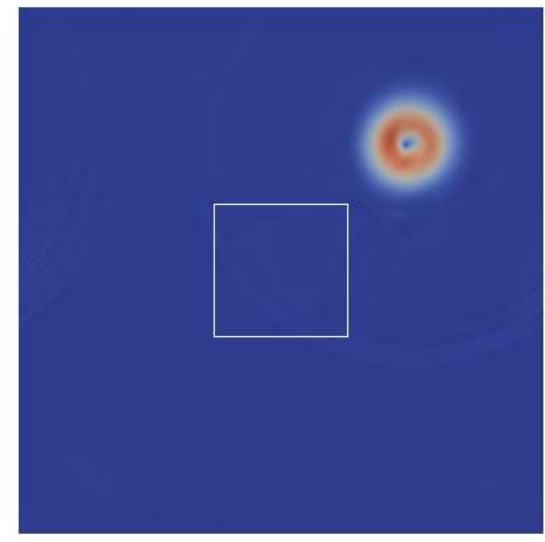

(d) Projection; $t=10$

Figure 8: Perturbation-velocity magnitude $\left|\mathbf{u}_{\mathrm{E}}-\mathbf{u}_{\infty}\right|$ in $\Omega_{\mathrm{E}}$ at $t=5$ and $t=10$ for two-way partial-boundary coupling and projection coupling. The domain $\Omega_{\mathrm{NS}}$ is outlined. 


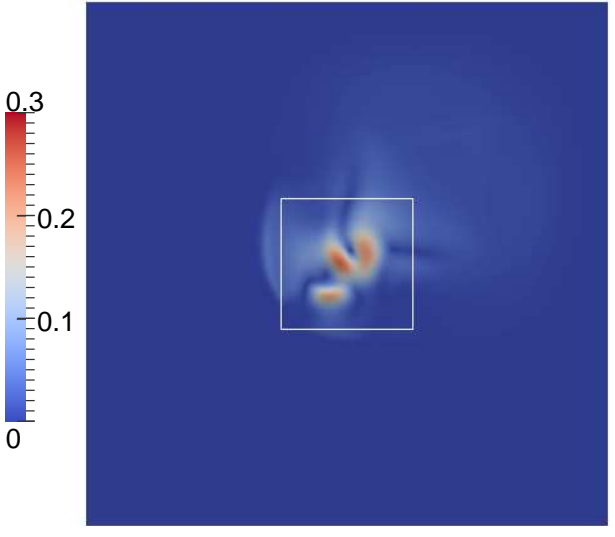

(a) Partial-boundary; $t=5$

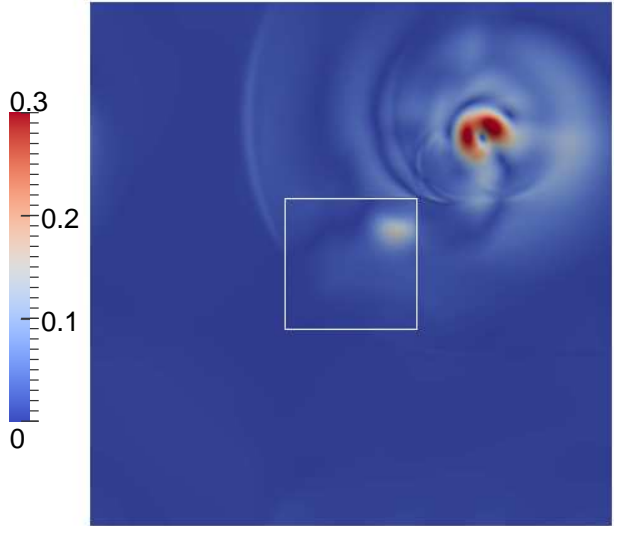

(c) Partial-boundary; $t=10$

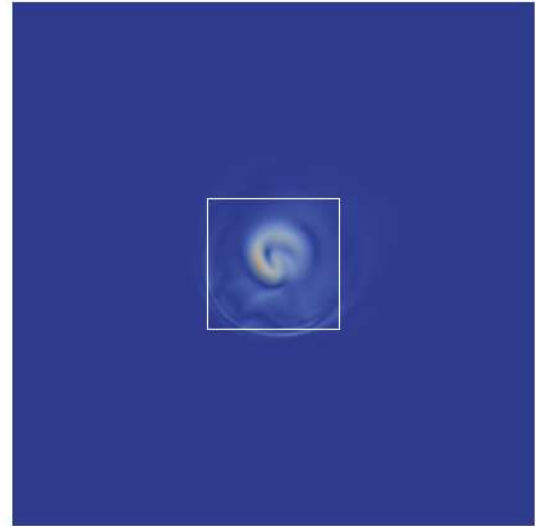

(b) Projection; $t=5$

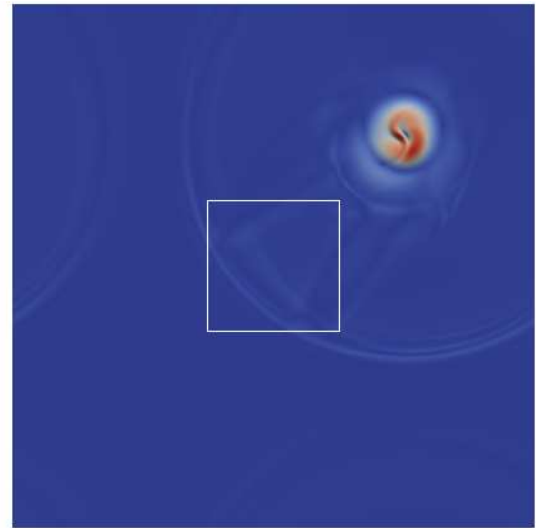

(d) Projection; $t=10$

Figure 9: Normalized velocity-deviation magnitude, $D\left(\mathbf{u}_{\mathrm{E}}\right)$, in $\Omega_{\mathrm{E}}$ at $t=5$ and $t=10$ for two-way partial-boundary coupling and projection coupling. The domain $\Omega_{\mathrm{NS}}$ is outlined. 
is much better than one-way coupling. Alternatively, for partial-boundary coupling, deviation is greater in two-way coupling than in one-way coupling and there is much greater late-time error in the NS domain.

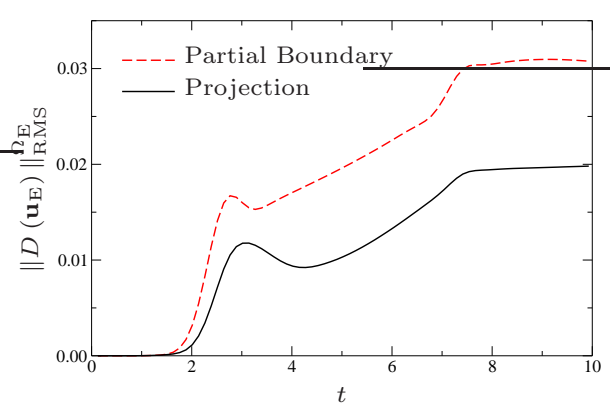

(a) Euler domain

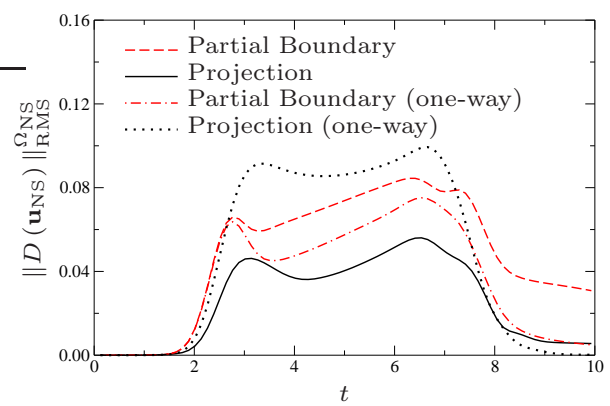

(b) NS domain

Figure 10: RMS of the normalized velocity deviation calculated over (a) $\Omega_{\mathrm{E}}$ and (b) $\Omega_{\mathrm{NS}}$ as a function of time under partial-boundary and projection two-way coupling. In (b), also included are the results for one-way coupling, c.f. Fig. 6.

As in one-way coupling, we examine the eddy kinetic energy in $0<t<10$. Figure 11(a) shows $\operatorname{EKE}\left(\Omega_{\mathrm{E}}, \mathbf{u}, \rho\right)$ for the Euler domain. Here, the uncoupled solution is constant. The coupled-solution histories show a slight increase in EKE when the vortex enters the NS domain, but then show expected decay due to viscous dissipation. Projection coupling shows the best late-time performance. Corresponding EKE histories are shown in Figure 11(b) for the NS domain, where we have included results for one-way coupling (c.f. Fig. 7). Here we see that both projection- and partial-boundary-coupling solutions are improved by going to two-way coupling, but projection coupling shows the best performance.

We are also interested in the performance of two-way projection coupling for smaller vortices. Figure 12 shows perturbation-velocity magnitude and 


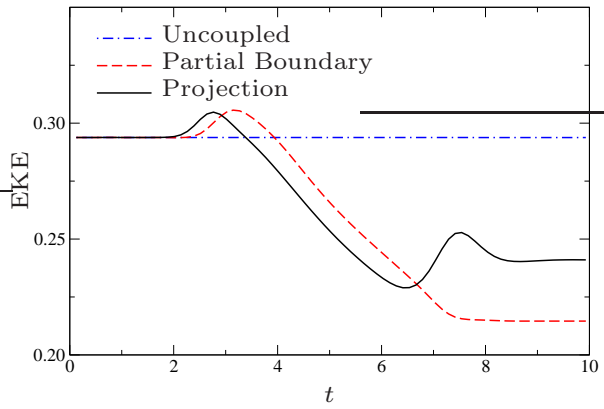

(a) Euler domain

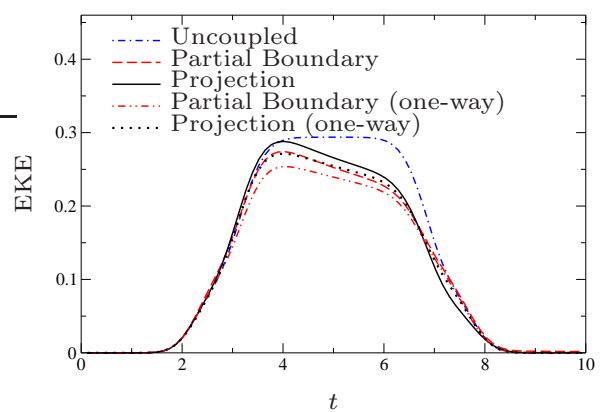

(b) NS domain

Figure 11: (a) Eddy kinetic energy in $\Omega_{\mathrm{E}}$ for the uncoupled Euler-domain solution $\left(\rho_{\mathrm{E}}^{\mathrm{UC}}, \mathbf{u}_{\mathrm{E}}^{\mathrm{UC}}\right)$ and the Euler-domain solution $\left(\rho_{\mathrm{E}}, \mathbf{u}_{\mathrm{E}}\right)$ under partial-boundary and projection two-way coupling. (b) Eddy kinetic energy in $\Omega_{\mathrm{NS}}$ for the uncoupled Euler-domain solution $\left(\rho_{\mathrm{E}}^{\mathrm{UC}}, \mathbf{u}_{\mathrm{E}}^{\mathrm{UC}}\right)$ and the NS-domain solutions $\left(\rho_{\mathrm{NS}}, \mathbf{u}_{\mathrm{NS}}\right)$ under partial-boundary and projection two-way coupling. Also included in (b) are the results for one-way coupling, c.f. Fig. 7.

the normalized velocity-deviation magnitude for a vortex with the same magnitude as the baseline case, but with a smaller area; the vortex is shown at $t=10$ after it has passed through the NS domain. Vortex parameters are $\eta=3$ and $\alpha=0.4688$ and are otherwise the same as in the baseline case. The numerical discretization parameters were also the same as in the baseline case, except for the number of acoustic substeps; six substeps were taken here for stability, whereas only two were required in the baseline case. We see that the vortex has decayed more so than in the baseline case after passing through the NS domain due to the steeper velocity gradients of the vortex. However, the vortex's structure is maintained well as in the baseline case and the scattered acoustic waves have even smaller amplitudes. 


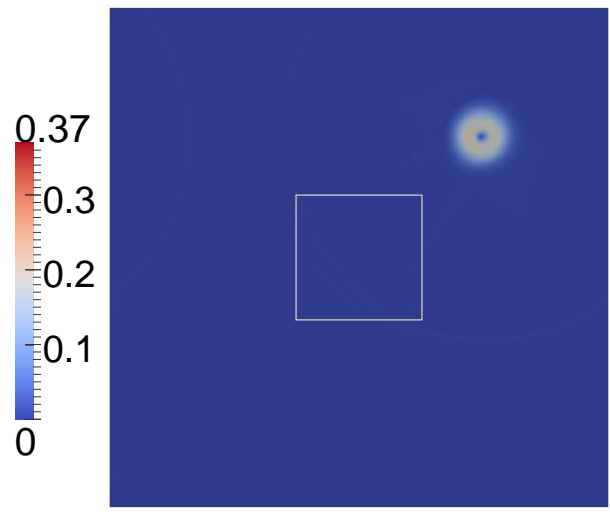

(a)

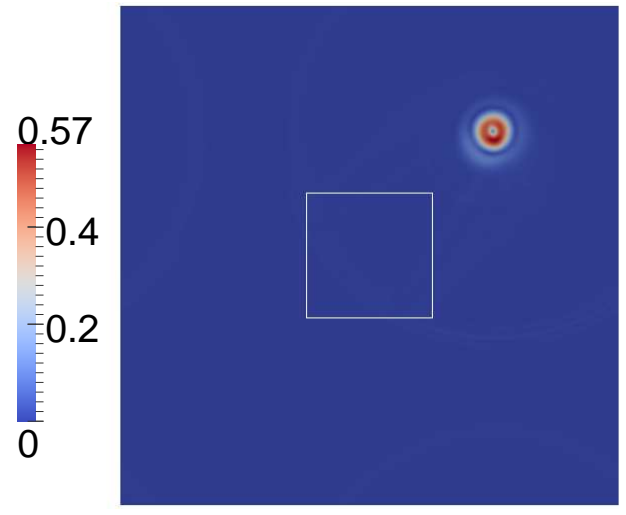

(b)

Figure 12: (a) Perturbation-velocity magnitude $\left|\mathbf{u}_{\mathrm{E}}-\mathbf{u}_{\infty}\right|$ and (b) normalized velocitydeviation magnitude, $D\left(\mathbf{u}_{\mathrm{E}}\right)$, in $\Omega_{\mathrm{E}}$ at $t=10$ for two-way projection coupling for the smaller vortex case. The domain $\Omega_{\mathrm{NS}}$ is outlined.

\subsection{Grid refinement}

We consider here the grid dependence of the solutions shown in the previous sections. In particular, we examine solutions calculated on a more refined grid: $1200 \times 1200$ and $300 \times 300$ grid points for $\Omega_{\mathrm{E}}$ an $\Omega_{\mathrm{NS}}$, respectively (compared with $400 \times 400$ and $100 \times 100)$. For one-way coupling, Figure 13 compares the velocity-deviation results of Figure 6 with those from the more refined grid. We see that the projection results are largely grid independent. However, the partial-boundary results are only grid independent for $t<6$. There is a larger discrepancy as the vortex leaves the NS domain through the North-East corner. For two-way coupling, Figure 14 compares the results of Figure 10 with those calculated with the refined model. For partial-boundary coupling, we again see an increase in the discrepancy as the vortex leave the NS domain. However, for projection coupling there is a slight decrease in the discrepancy within $\Omega_{\mathrm{E}}$ after the vortex leaves the domain $\Omega_{\mathrm{NS}}$. Having 
the vortex exit through a mesh corner makes this a worst-case scenario for this system. Overall, we see that the solutions calculated with projection coupling are much less sensitive to grid resolution than those calculated with partial-boundary coupling.

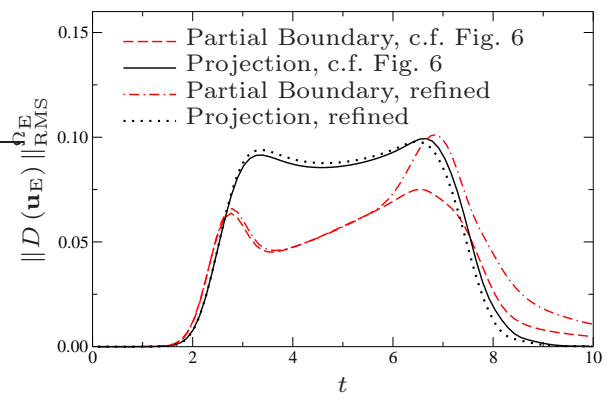

Figure 13: RMS of the normalized velocity deviation calculated over $\Omega_{\mathrm{NS}}$ as a function of time under partial-boundary and projection one-way coupling. Results are shown from Fig. 6 and those from a more refined grid.

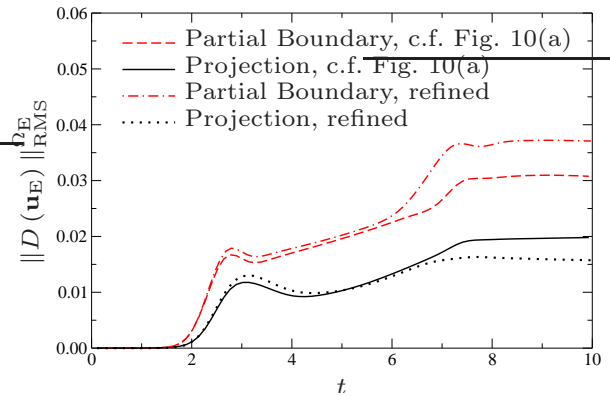

(a) Euler domain

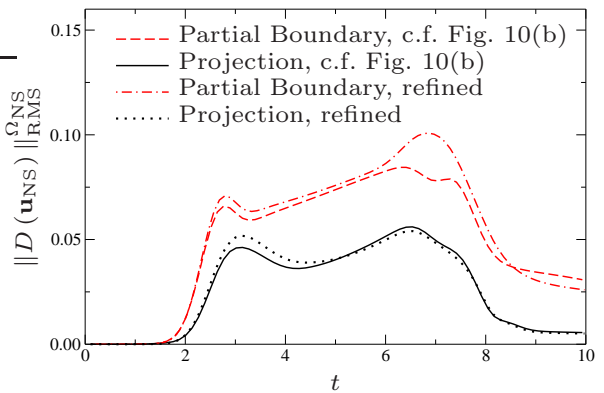

(b) NS domain

Figure 14: RMS of the normalized velocity deviation calculated over (a) $\Omega_{\mathrm{E}}$ and (b) $\Omega_{\mathrm{NS}}$ as a function of time under partial-boundary and projection two-way coupling. Results are shown from Fig. 10 and those from a more refined grid. 


\section{Conclusion}

In this paper we introduced two new schemes for one- and two-way coupling where a meso-scale NWP code drives a micro-scale CFD code: partialboundary coupling and projection coupling. Variations on partial-boundary coupling have have been implemented elsewhere in one-way coupling in realistic flow conditions with limited success; two-way coupling has remained elusive. We implemented our coupling schemes in a simplified two-dimensional system, for which we could better understand and interrogate couplingmethod performance. Our meso-scale model was the inviscid compressible Euler equations; our model neglected the many physics models required for accurate weather simulation. Such models include, for example, those for cloud physics, convective parameterization, and turbulence. Our micro-scale model was the incompressible Navier-Stokes equations. Numerical methods for solving these systems mimicked the WRF-NWP and OpenFOAM-CFD codes, respectively. Our test case was a propagating isentropic vortex in laminar flow; an exact solution to the compressible Euler equations. The driving question was: Can we propagate a simple laminar vortex from one domain to another in a physically relevant manner, given that the domains have different mathematical and numerical-discretization models? This work was designed as a first step towards accurate coupling of fully turbulent flows between such codes in three dimensions.

In both partial-boundary and projection coupling, the vortex was coherently passed between domains in one- and two-way coupled configurations. In one-way coupling, partial-boundary coupling performed better than projection coupling in that there was less deviation from the uncoupled Euler- 
domain solution. The reverse was true in two-way coupling. Perhaps most impressive was that, in two-way coupling, projection coupling well maintained the vortex structure in both the meso- and micro-scale domains. Both coupling approaches show promise for extension to three-dimensional flows.

Looking forward to coupling three-dimensional turbulent flow, several challenges are anticipated. First and foremost, it will be challenging to properly transfer grid-scale and subgrid-scale turbulence between domains, which will likely have different turbulence models. Second, spatial and temporal grids in realistic simulations will be non-matching. The simulations described here employed grids that matched in space and time; laminar-flow simulations on nonmatching grids can be accomplished through consistent interpolation and similar performance is expected. Non-matching grids, however, may present a significant problem in three-dimensional turbulent flow, where LES turbulence models can be significantly affected by grid size. This will be further complicated by the grids in a code like WRF, which move in the vertical direction as a function of pressure.

\section{Acknowledgement}

The authors acknowledge useful discussions with Matt Churchfield, Julie Lundquist, Pat Moriarty, and John Michalakes, and feedback from the reviewers. This work was supported by the U.S. Department of Energy under Contract No. DE-AC36-08-GO28308 with the National Renewable Energy Laboratory. Funding was provided in part by a grant from the Center for Research and Education in Wind (CREW), and the NREL Laboratory Directed Research and Development (LDRD) program. 


\section{References}

[1] Complex Flow Workshop Report, January 17-18, 2012, University of Colorado, Boulder, US Department of Energy, Energy Efficiency and Renewable Energy, Wind Program, 2012.

[2] R. J. Barthelmie, L. E. Jensen, Evaluation of wind farm efficiency and wind turbine wakes at the Nysted offshore wind farm, Wind Energy 13 (2010) 573-586.

[3] M. J. Churchfield, S. Lee, M. J. Moriarty, L. A. Martinez, S. Leonardi, G. Vijayakumar, J. G. Brasseur, A large-eddy simulation of wind-plant aerodynamics, in: Proceedings of the 50th AIAA Aerospace Sciences Meeting, Nashville, TN, 2012.

[4] W. Musial, S. Butterfield, B. McNiff, Improving wind turbine gearbox reliability, in: Proceedings of the 2007 European Wind Energy Conference, Milan, Italy, 2007.

[5] M. A. Sprague, P. J. Moriarty, M. J. Churchfield, K. Gruchalla, S. Lee, J. Lundquist, J. Michalakes, A. Purkayastha, Computational modeling of wind-plant aerodynamics, in: Proceedings of SciDAC 2011, 2011.

[6] W. C. Skamarock, J. B. Klemp, J. Dudhia, G. Gill, D. Barker, W. Wang, J. G. Powers, A Description of the Advanced Research WRF Version 2, Technical Report NCAR/TN-468+STR, NCAR, 2005.

[7] OpenFOAM - The Open Source CFD Toolbox, User's Manual, Version 1.6, OpenCFD Ltd, Berkshire, UK, 2009. 
[8] W. J. Coirier, S. Kim, S. Marella, J. Mayes, F. Chen, J. Michalakes, S. Miao, Progress towards a coupled mesoscale and microscale modeling capability, in: Proceedings of the 7th Symposium on the Urban Environment, 2007.

[9] L. Li, L.-J. Zhang, N. Zhang, F. Hu, Y. Jiang, C.-Y. Xuan, W.-M. Jiang, Study on the micro-scale simulation of wind field over complex terrain by RAMS/FLUENT modeling system, in: Proceedings of the Fifth International Symposium on Computational Wind Engineering (CWE2010), Chapel Hill, NC, 2010.

[10] Z. Boutanios, C. Miller, H. Hangan, Computational analysis of the Manitoba September 51996 storm: mesoscale WRF-ARW simulations coupled with microscale OpenFOAM CFD simulations, 2010. Talk at The Fifth International Symposium on Computational Wind Engineering, Chapel Hill, North Carolina, May 23-27.

[11] F. Zajaczkowski, S. Haupt, K. Schmehl, A preliminary study of assimilating numerical weather prediction data into computational fluid dynamics models for wind prediction, Journal of Wind Engineering and Industrial Aerodynamics 99 (2011) 320-329.

[12] AcuSolve Command Reference Manual, Version 1.8, ACUSIM Software, 2008.

[13] M. J. Churchfield, M. A. Sprague, J. Michalakes, B. Vanderwende, S. Lee, C. Draxl, J. K. Lundquist, A. Purkayastha, P. J. Moriarty, Using mesoscale weather model output as boundary conditions for at- 
mospheric large-eddy simulations and wind-plant aerodynamics simulations, in: Proceedings of International Conference on Future Technologies for Wind Energy, Laramie, WY, 2013.

[14] J. Sitaraman, D. Mavriplis, E. P. Duque, Wind farm simulations using a full rotor model for wind turbines, in: Proceedings of the AIAA SciTech 2014 Meeting, National Harbor, MD, 2014.

[15] V. Sankaran, J. Sitaraman, A. Wissink, B. Jayaraman, A. Datta, Z. Yang, D. Mavriplis, H. Saberi, M. Potsdam, D. O'Brien, R. Cheng, N. Hariharan, R. Strawn, Application of the HELIOS computational platform to rotorcraft flowfields, in: Proceedings of the 48th AIAA Aerospace Sciences Meeting, Orlando, FL, 2010.

[16] H. Gopalan, C. J. S. W. M. Gundling, J. Mirocha, Coupled mesoscale microscale model for wind resource estimation and turbine aerodynamics using an overset grid approach, in: Proceedings of the 51st AIAA Aerospace Sciences Meeting and Exhibit, Dallas, TX, 2013.

[17] Yang, X., F. Sotiropoulos, R. J. Conzemius, J. N. Wachtler, and M. B. Strong (2014). Large-eddy simulation of turbulent flow past wind turbines/farms: the Virtual Wind Simulator (VWiS). Wind Energy.

[18] T. Yamada, K. Koike, Downscaling mesoscale meteorological models for computational wind engineering applications, Journal of Wind Engineering and Industrial Aerodynamics 99 (2011) 199-216.

[19] Y. V. Peet, S. K. Lele, Computational framework for coupling compressible and low Mach number codes, AIAA Journal 46 (2008) 1990-2001. 
[20] Y. V. Peet, S. K. Lele, Near field of film cooling jet issued into a flat plate boundary layer: LES study, in: Proceedings of ASME Turbo Expo 2008, Berlin, Germany, 2008.

[21] P. P. Sullivan, J. C. McWilliams, C.-H. Moeng, A grid nesting method for large-eddy simulation of planetary boundary-layer flows, BoundaryLayer Meteorology 80 (1996) 167-202.

[22] C.-H. Moeng, J. Dudhia, J. Klemp, P. Sullivan, Examining two-way grid nesting for large eddy simulation of the PBL using the WRF model, Monthly Weather Review 135 (2007) 2295-2311.

[23] J. Mirocha, G. Kirkil, E. Bou-Seid, F. K. Chow, B. Kosović, Transition and equilibration of neutral atmospheric boundary layer flow in one-way nested large eddy simulations using the weather research and forecasting model, Monthly Weather Review 141 (2013) 918-940.

[24] J. Mirocha, B. Kosović, G. Kirkil, Resolved turbulence characteristics in large-eddy simulations nested within mesoscale simulations using the Weather Research and Forecasting model, Monthly Weather Review 142 (2014) 806-831.

[25] L. M. Harris, D. R. Durran, An idealized comparison of one-way and two-way grid nesting, Monthly Weather Review 138 (2010) 2174-2187.

[26] C. A. Felippa, K. C. Park, C. Farhat, Partitioned analysis of coupled mechanical systems, Computer Methods in Applied Mechanics and Engineering 190 (2001) 3247-3270. 
[27] L. J. Wicker, W. C. Skamarock, Time-splitting methods for elastic models using forward time schemes, Monthly Weather Review 130 (2002) 2088-2097.

[28] F. M. Mesinger, Forward-backward scheme, and its use in a limited area model, Contrib. Atmos. Phys. 50 (1977) 200-210.

[29] C. M. Rhie, W. L. Chow, Numerical study of the turbulent flow past an airfoil with trailing edge separation, AIAA Journal 21 (1983) 1525-1532.

[30] R. I. Issa, Solution of the implicitly discretized fluid flow equations by operator-splitting, Journal of Computational Physics 62 (1985) 40-65.

[31] E. Garnier, P. Sagaut, M. Deville, A class of explicit ENO filters with application to unsteady flows, Journal of Computational Physics 170 (2001) 184-201.

[32] Y. C. Zhou, G. W. Wei, High resolution conjugate filters for the simulation of flows, Journal of Computational Physics 189 (2003) 159-179. 